\title{
Clostridium septicum aortitis with synchronous ascending colon and rectal adenocarcinoma
}

\author{
Deepanshu Jain ${ }^{a}$, Andrew C. Kistlerb, Patricia Kozuch ${ }^{\mathbf{b}}$ \\ Albert Einstein Medical Center; Thomas Jefferson University Hospital, Philadelphia, USA
}

\begin{abstract}
Clostridium septicum (C. septicum) aortitis is a rare condition frequently associated with colon adenocarcinoma and carries a poor prognosis. We report the case of a 66-year-old man who presented with abdominal pain, blood in the stool, fever and chills. Laboratory tests were significant for leukocytosis and microcytic anemia. Abdominal imaging revealed a right colon mass and aortitis. Colonoscopy confirmed the right colon mass and also discovered a rectal mass, both adenocarcinomas. Treatment consisted of antibiotics, aortic repair, right hemi-colectomy and later trans-anal excision of the rectal mass. Blood cultures and the aortic specimen grew C. septicum. The patient improved and was doing well in follow up.
\end{abstract}

Keywords Clostridium septicum, aortitis, colorectal adenocarcinoma

Ann Gastroenterol 2017; 30 (4): 468-470

\section{Introduction}

Sepsis secondary to bacterial species such as Streptococcal bovis [1] and Clostridium septicum (C. septicum) [2] is associated with occult colorectal malignancy. C. septicum is a gram-positive spore that forms an obligate anaerobic rod. The colon may be a preferential site of growth for this organism based on the $\mathrm{pH}$ (5.5-7.0) of the large intestine and its resulting osmotic environment [3]. C. septicum sepsis can present as vasculitis [4], mycotic aneurysm [5], or pericarditis [6], and has a high mortality [2]. We present a case of C. septicum aortitis associated with colonic malignancy, along with its management and clinical outcome.

\section{Case report}

A 66-year-old male, originally from Puerto Rico, with no significant past medical history presented with three days of

Division of Gastroenterology and Hepatology, Department of Medicine aAlbert Einstein Medical Center (Deepanshu Jain); ${ }^{\text {b} T h o m a s ~ J e f f e r s o n ~}$

University Hospital (C. Andrew Kistler, Patricia Kozuch), Philadelphia, USA

Conflict of Interest: None

Correspondence to: Deepanshu Jain, MD, Gastroenterology and Hepatology, Department of Internal Medicine, Albert Einstein Medical Center, Philadelphia, PA, 19141, USA, Tel.: +1 2154568520 ,

Fax: +1 215456 7926, e-mail: deepanshu.jain.25@gmail.com

Received 1 February 2017; accepted 28 February 2017; published online 28 March 2017

DOI: https://doi.org/10.20524/aog.2017.0140 colicky upper abdominal pain associated with one episode of bright red blood mixed with stool. He reported subjective fevers with chills. He had new-onset intermittent constipation during the previous month. He denied nausea, vomiting, diarrhea, pruritus, weight loss, fatigue or jaundice. Family history was negative for colon cancer. He had no history of smoking or alcohol abuse; further, he denied any high-risk sexual behavior or any history of or exposure to tuberculosis and syphilis.

The patient presented to an outside hospital with stable vital signs except for being febrile $\left(38.3^{\circ} \mathrm{C}\right)$. Lab results showed leukocytosis and anemia. This was followed by an abdominal ultrasound that was suspicious for right colon mass and infrarenal aortitis. The patient was started on broad-spectrum antibiotics and transferred to our hospital. On arrival he was febrile to $38.9^{\circ} \mathrm{C}$, but remained hemodynamically stable. Initial blood tests here showed white blood cells 19,500 cells $/ \mu \mathrm{L}$ with $87 \%$ neutrophils, and hemoglobin $9.0 \mathrm{~g} / \mathrm{dL}$ with a mean corpuscular volume $71 \mathrm{fL}$. HIV, liver enzymes, carcino-embryonic antigen and lipase were within normal limits. Computed tomography (CT) of the abdomen and pelvis confirmed infrarenal aortitis and a right colon mass (Fig. 1A,B).

Colonoscopy revealed a $2-\mathrm{cm}$ mass in the proximal ascending colon (Fig. 2A), as well as a $5-\mathrm{cm}$ rectal mass (Fig. 2B). While both masses were grossly suspicious for malignancy, the rectal mass biopsy revealed only high-grade dysplasia. The right colon mass was not biopsied because a right hemicolectomy was already planned for the same day.

The patient underwent transabdominal excision of the infected infrarenal abdominal aorta, as well as the proximal bilateral common iliac arteries and proximal inferior mesenteric artery, followed by reconstruction with aorto-iliac cryopreserved homograft and left renal artery re-implantation. 


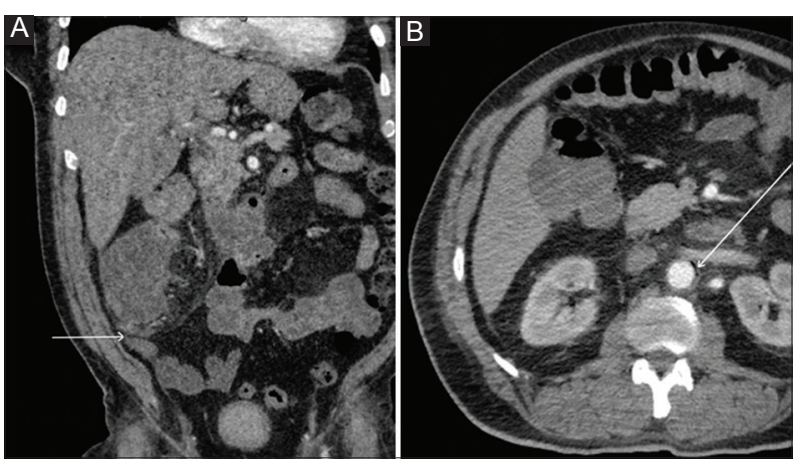

Figure 1 White arrows point to the right colon mass (A) and periaortic gas (B) seen on computed tomography scan

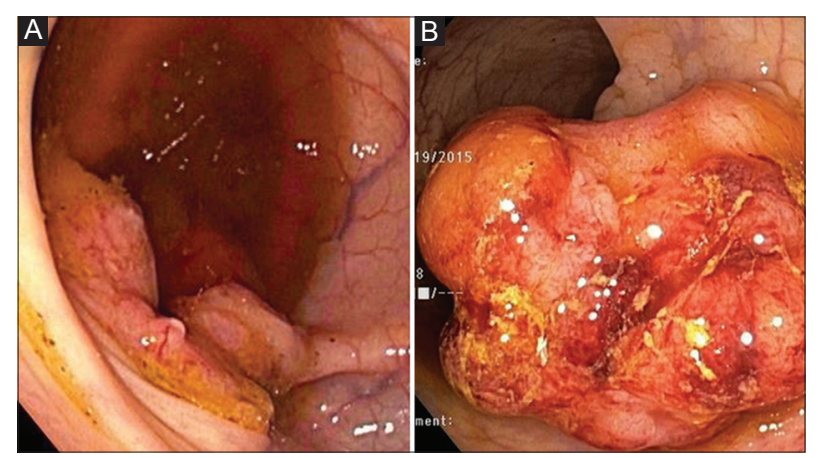

Figure 2 Right colon mass (A) and rectal mass (B) on colonoscopy

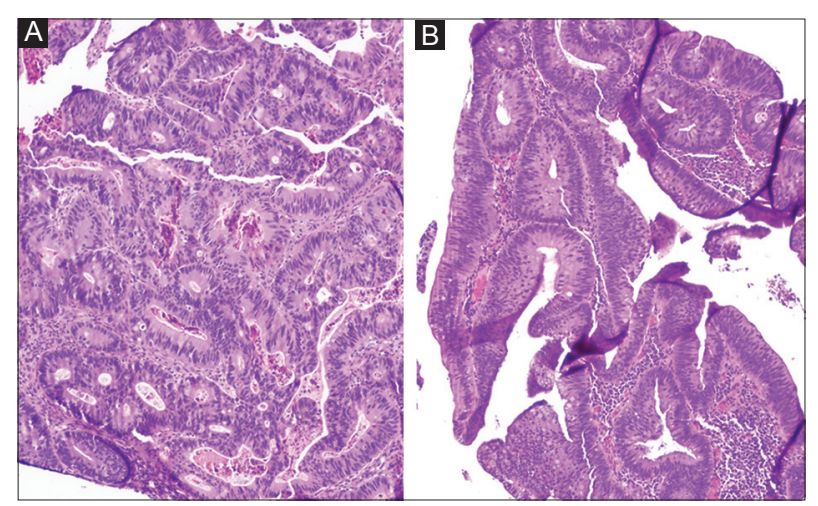

Figure 3 Histology of right colon mass (A) and rectal mass (B)

An extended right hemicolectomy and end ileostomy were also performed.

Cultures from blood and from the aorta grew C. septicum, sensitive to ampicillin-sulbactam. The right colon mass was an adenocarcinoma (Fig. 3A) (T3N0), negative for deficient mismatch repair protein expression, B-RAF (B-type rapidly accelerated fibrosarcoma) and K-RAS (Kirsten rat sarcoma oncogene) mutation but positive for N-RAS (neuroblastoma RAS oncogene).

The patient was treated with IV ampicillin-sulbactam and improved clinically, with subsequent negative blood cultures. He was discharged four weeks after admission and completed an additional two weeks of IV antibiotics. Follow-up positron emission tomography CT scan revealed a focus of nonspecific increased metabolic activity in the peritoneal cavity and mesenteric lymph nodes. Three months later, after full recovery from the surgery and sepsis, the patient underwent trans-anal intramural excision of the rectal mass, which was an invasive adenocarcinoma on histology but negative for C. septicum. Tumor-free margins were achieved (Fig. 3B). The tumor was negative for deficient mismatch repair protein expression, B-RAF and N-RAS mutation, but positive for K-RAS. The patient had a low oncotype DX recurrence score (11) and was offered but declined adjuvant chemotherapy.

\section{Discussion}

This case represents the first case of C. septicum aortitis in the setting of synchronous colon and rectal adenocarcinoma. Infection with this organism may occur in the presence of trauma, but commonly presents without trauma. A review conducted by Kornbluth et al reported that, out of 162 cases of atraumatic C. septicum infection, 23 patients had aortitis. Of these 23 patients, 21 (91\%) had colonic adenocarcinoma or polyps, while 15 of the 21 (71\%) had cancer of the ascending colon or cecum [7], suggestive of a strong association between right sided colon cancer and this infection.

Although C. septicum was initially thought to be a member of the normal gut flora, recent evidence from healthy volunteer stool studies may suggest the opposite [8]. Mildly acidic $\mathrm{pH}$ and a low propensity for oxidation-reduction reactions, as provided by cecal tumors, is a perfect milieu for the C. septicum to flourish. Its flagella and the ability to produce multiple enzymes allow it to grow within the host [9]. The virulence of C. septicum comes from its alpha toxin, a hemolysin [2].

The 6-month mortality for C. septicum aortitis has been reported to be as high as $100 \%$ in the absence of operative intervention, but can be reduced to $64 \%$ with appropriate surgical intervention [10]. The mortality is even higher among patients who present with distant myonecrosis (infection distant from the initial site of infection) [7]. This underscores the importance of searching for underlying malignancy in the setting of C. septicum aortitis.

All patients with suspected aortitis should be treated with broad-spectrum antibiotics while awaiting blood culture results. The antibiotic of choice for $C$. septicum infection is high-dose intravenous penicillin $\mathrm{G}$ [10], which should be given for a minimum of 6-8 weeks. Few patients require chronic antimicrobial therapy for severe infection or inability to control the source of infection. Early debridement and reconstruction of the aorta is essential, to prevent progression to aneurysm and aortic dissection [10].

Our patient underwent prompt source control for the infection and aortic reconstruction with appropriate antibiotic coverage for presumed C. septicum infection, which resulted in prompt recovery and a good prognosis. The finding of a synchronous lesion in the rectum raised a few interesting questions, including whether the rectal mass could have been the source of infection, and thus consideration for a total proctocolectomy and end ileostomy. After a review of 
the literature and a multidisciplinary discussion between the vascular and general surgeons, infectious disease specialists and gastroenterologists, it was thought that the right colon mass was more likely to be the culprit lesion; it was therefore resected at the same time as the aortic reconstruction. More experience with such an atypical scenario is required to make a better consensus treatment plan in the future for C. septicum aortitis associated with colon cancer, with the intent of improving outcomes and prognosis.

\section{References}

1. Abdulamir AS, Hafidh RR, Abu Bakar F. The association of Streptococcus bovis/gallolyticus with colorectal tumors: the nature and the underlying mechanisms of its etiological role. J Exp Clin Cancer Res 2011;30:11.

2. Kennedy CL, Krejany EO, Young LF, et al. The alpha-toxin of Clostridium septicum is essential for virulence. Mol Microbiol 2005;57:1357-1366.
3. Koransky JR, Stargel MD, Dowell VR Jr. Clostridium septicum bacteremia. Its clinical significance. Am J Med 1979;66:63-66.

4. Shah S, Whitehead D, Sampath K, Toor A. A case of Clostridium septicum aortitis with concomitant adenocarcinoma of the cecum. ACG Case Rep J 2015;2:230-232.

5. Lintin L, Wheeler R, Whiston R, Gordon A, Berry D, Torkington J. Mycotic thoracic aortic arch aneurysm from haematogenous spread of Clostridium septicum due to metastatic colorectal cancer: a survival guide. J Surg Case Rep 2014;2014: rju117. doi: 10.1093/ jscr/rjul17.

6. Pigrau C, Ruiz MP, Sagrista J. Purulent pericarditis due to Clostridium septicum associated with carcinoma of the colon. Clin Infect Dis 1995;20:202-203.

7. Kornbluth AA, Danzig JB, Bernstein LH. Clostridium septicum infection and associated malignancy. Report of 2 cases and review of the literature. Medicine (Baltimore) 1989;68:30-37.

8. Moore WE, Holdeman LV. Human fecal flora: the normal flora of 20 Japanese-Hawaiians. Appl Microbiol 1974;27:961-979.

9. Bernheimer AW. Parallelism in the lethal and hemolytic activity of the toxin of Clostridium septicum. J Exp Med 1944;80:309-320.

10. Seder CW, Kramer M, Long G, Uzieblo MR, Shanley CJ, Bove P. Clostridium septicum aortitis: report of two cases and review of the literature. J Vasc Surg 2009;49:1304-1309. 